\title{
Database of oceanographic anomalies and atmospheric surface fluxes for the study of climate change in the Brazilian Northeast.
}

\author{
Humberto L. Varona ${ }^{* 1}$; Moacyr Araujo ${ }^{1}$, Marcus Silva ${ }^{1}$, Mauro Maida ${ }^{1}$, Beatrice P. Ferreira ${ }^{1}$
}

\begin{abstract}
This paper presents three datasets of anomalies of oceanic physical parameters and surface atmospheric fluxes in the northeast region of Brazil, geographically limited by $42.5^{\circ} \mathrm{W}-29.75^{\circ} \mathrm{W} / 20.5^{\circ} \mathrm{S}-1.25^{\circ} \mathrm{N}$, calculated from the SODA, TROPFLUX and GPCP datasets. The DASAF-BNE (Daily Anomalies of the Surface Atmospheric Fluxes of the Brazilian Northeast), DRA-BNE (Daily Rainfall Anomalies in the Brazilian Northeast), and MAOP-BNE (Monthly Anomalies of Oceanographic Parameters in the Brazilian Northeast) datasets are generated with $0.25^{\circ}$ resolution bilinearly interpolating the original datasets. This database includes four files in NetCDF format, which facilitates its handling due to the diversity of freeware tools that exist, DASAF-BNE and DRA-BNE have a daily frequency, while MAOP-BNE has a monthly frequency. This database is mainly intended to research climate changes in the Brazilian Northeast.
\end{abstract}

Keywords: SODA; TROPFLUX; GPCP; Anomalies; Brazilian Northeast.

\section{DATA IMPORTANCE}

- This dataset is made up of multiple NetCDF files, making it easy to share, is extremely easy to use, and does not require any prior processing.

- The data presented here cover physical parameter anomalies in the northeastern region of Brazil and may be useful to any researcher who may need these data for further analysis or to interpret physical patterns or processes.

- These data are relevant for studies of climate changes in the northeastern region of Brazil and can be reused for statistical studies of coastal and oceanic climate variations.

\footnotetext{
${ }^{1}$ Federal University of Pernambuco, Department of Oceanography, Recife-PE, Brazil. (Humberto.varona@yandex.com)
} 


\section{MATERIALS AND METHODS}

This dataset presents the anomalies of ocean physical parameters and surface atmospheric fluxes obtained from the SODA (Simple Ocean Data Assimilation, CARTON; GIESE, 2008), GPCP (Global Precipitation Climatology Project, ADLER et al., 2003), and TROPFLUX datasets (Air-Sea Fluxes for the Global Tropical Oceans, KUMAR et al., 2011). The anomalies of all the parameters of these three datasets were calculated and reduced to the limits $42.5^{\circ} \mathrm{W}-29.75^{\circ} \mathrm{W} / 20.5^{\circ} \mathrm{S}-1.25^{\circ} \mathrm{N}$ (Fig. 1), which corresponds to the northeastern region of Brazil. All files in these three datasets are in standard NetCDF format. The time reference of these datasets was standardized with the same time offset (days since 1900-1-1 00:00:00) and for all parameters, the missing data is represented by NaN (Not a Number), in the metadata of each parameter_fillValue and missing_value are assigned to $\mathrm{NaN}$.

All files were created with the Matlab script set mNC of Varona (2021a) after being bilinearly interpolated to $0.25^{\circ}$ resolution with the Matlab function griddata. Using the Matlab script set CalcPlotAnomaly (VARONA, 2021b) the anomaly calculations were performed. The freeware programs ncview (visual browser for netCDF, PIERCE, 2016), ncdump (Unidata's Utility for viewing the NetCDF file structure), nco (netCDF operators, ZENDER, 2008) and CDO (Climate Data Operator, SCHULZWEIDA, 2006) were used for pre-and post-processing (metadata creation) of all datasets.

Table 1 shows the characteristics of the original and created datasets: DASAF-BNE (Daily Anomalies of the Surface Atmospheric Fluxes of the Brazilian Northeast), DRA-BNE (Daily Rainfall Anomalies in the Brazilian Northeast), and MAOPBNE (Monthly Anomalies of Oceanographic Parameters in the Brazilian Northeast), as well as, the names of the files corresponding to each of these datasets. The time period, frequency and resolution of the data are also shown.
Figure 1. Geographical location of the datasets presented in this datapaper $\left(42.5^{\circ} \mathrm{W}-29.75^{\circ} \mathrm{W} / 20.5^{\circ} \mathrm{S}-1.25^{\circ} \mathrm{N}\right)$, the bathymetry of the region and the study region.

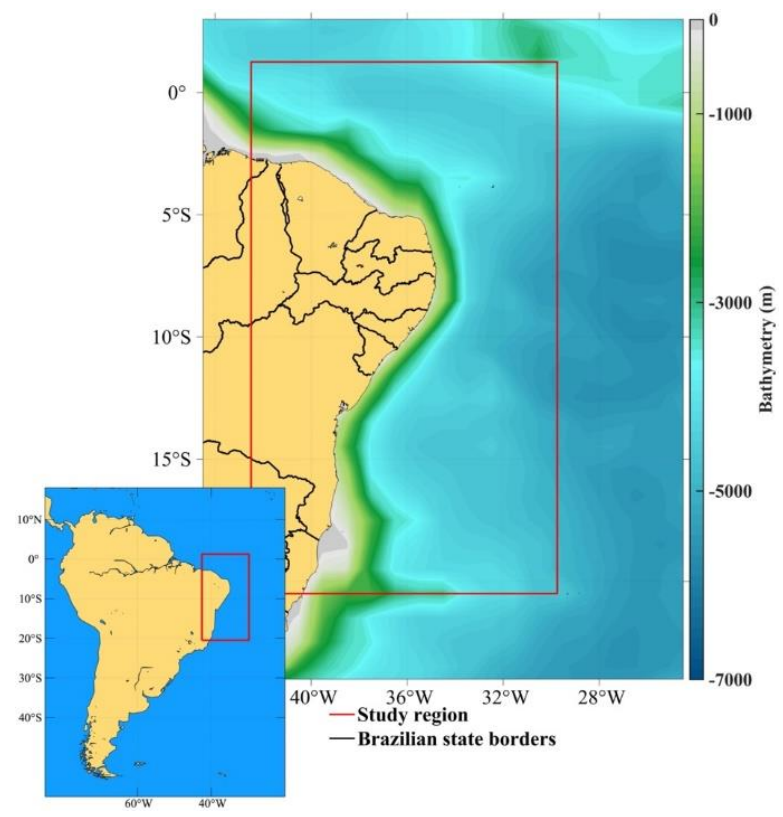

\section{DATA DESCRIPTION}

The dataset presented here are 4 files in NetCDF format, these files contain the physical parameter anomalies calculated from the SODA, TROPFLUX and GPCP datasets. The NetCDF files are self-descriptive, so by reading the metadata in the structure of each file you can know the names of the parameters, their description, unit of measurement, as well as the time offset value and the missing data value.

\section{Dataset}

The file anom_TROPFLUX_NE_CIMARII.nc (DASAF-BNE dataset) encompass the surface atmospheric flux anomalies calculated from the daily mean values of the parameters of the TROPFLUX dataset. In the DRA-BNE dataset, there is a single file named anom_Precip_NE_CIMARII.nc which contains the anomalies of the mean values of the precipitation accumulated in one day. The MAOP-BNE dataset consists of two files, anom_uvSODA_NE_CIMARII.nc contains the anomalies of the zonal and meridional 
components and the current velocity modulus, as well as the zonal and meridional components of the wind stress, the second file, named anom_OSODA_NE_CIMARII.nc contains the anomalies of the remaining parameters from the SODA dataset, including the vertical velocity of the currents.

The reason the MAOP-BNE dataset is divided into two files is that the horizontal components of ocean currents and wind stress have a different coordinate system (longitude and latitude) than the rest of the parameters. Table 2 shows the short name of each parameter, the standard name, and the units of measurement, as well as, the grid types for each of the anomaly datasets presented here. The grid-type defines the location of each data in the structure of each parameter, 4D type grids are organized similarly to 3D type grids, but in addition to horizontal coordinates and time (lon, lat, time) they also depend on depth (lon, lat, depth, time), lon and lat are the longitudes and latitudes respectively where each data is geographically located. The parameter wt (vertical velocity of the currents), has a different vertical coordinate system than the rest of the parameters, depending on wt(lat, lon, vdepth, time), where vdepth is the vertical coordinate for $w t$ and depth is the vertical coordinate for the rest of the parameters.

Table 1. Characteristics of each dataset created: DASAF-BNE (Daily Anomalies of the Surface Atmospheric Fluxes of the Brazilian Northeast), DRA-BNE (Daily Rainfall Anomalies in the Brazilian Northeast), and MAOP-BNE (Monthly Anomalies of Oceanographic Parameters in the Brazilian Northeast).

\begin{tabular}{ccccccc}
\hline Datasource & $\begin{array}{c}\text { Final } \\
\text { dataset }\end{array}$ & Time range & Frequency & $\begin{array}{c}\text { Original } \\
\text { resolution }\end{array}$ & $\begin{array}{c}\text { Final } \\
\text { resolution }\end{array}$ & Filename \\
\hline $\begin{array}{c}\text { TROPFLUX } \\
\text { (KUMAR et } \\
\text { al., 2011) }\end{array}$ & $\begin{array}{c}\text { DASAF } \\
\text { BNE }\end{array}$ & $\begin{array}{c}\text { From } \\
\text { Jan/1979 } \\
\text { to Dec/2017 }\end{array}$ & Daily & $1^{\circ}$ & $0.25^{\circ}$ & anom_TROPFLUX_NE_CIMARII.nc \\
$\begin{array}{c}\text { GPCP (ADLER } \\
\text { et al., 2003) }\end{array}$ & $\begin{array}{c}\text { DRA } \\
\text { BNE }\end{array}$ & $\begin{array}{c}\text { From } \\
\text { Oct/1996 } \\
\text { to Dec/2020 }\end{array}$ & Daily & $1^{\circ}$ & $0.25^{\circ}$ & anom_Precip_NE_CIMARII.nc \\
$\begin{array}{c}\text { SODA } \\
\text { (CARTON } \\
\text { and GIESE, }\end{array}$ & MAOP & $\begin{array}{c}\text { From } \\
\text { Jan/1980 }\end{array}$ & Monthly & 0.5 & 0.25 & anom_oSODA_NE_CIMARII.nc \\
\hline
\end{tabular}

\section{SUPPLEMENTARY MATERIALS}

Data deposited in recognized open repositories

Monthly Anomalies of Oceanographic Parameters in the Brazilian Northeast (MAOP-BNE).

Repository name: Zenodo

DOI of the dataset: https://doi.org/10.5281/zenodo.5559266

Link to access the data: https://zenodo.org/record/5559266

Daily Rainfall Anomalies in the Brazilian Northeast (DRA-BNE).

Repository name: Zenodo

DOI of the dataset: https://doi.org/10.5281/zenodo.5813779

Link to access the data: https://zenodo.org/record/5813779 
Daily Anomalies of the Surface Atmospheric Fluxes in the Brazilian Northeast (DASAF-BNE).

Repository name: Zenodo

DOI of the dataset: https://doi.org/10.5281/zenodo.5559230

Link to access the data: https://zenodo.org/record/5559230

Table 2. Description of parameters of the DASAF-BNE (Daily Anomalies of the Surface Atmospheric Fluxes of the Brazilian Northeast), DRA-BNE (Daily Rainfall Anomalies in the Brazilian Northeast), and MAOP-BNE (Monthly Anomalies of Oceanographic Parameters in the Brazilian Northeast) datasets.

\begin{tabular}{|c|c|c|c|c|}
\hline Parameter name & Description & Unit & Grid type & Dataset \\
\hline Ihf & Latent heat flux & $W m^{-2}$ & $3 D$ & DASAF-BNE \\
\hline Iwr & Net surface longwave radiation & $\mathrm{W} \mathrm{m}^{-2}$ & $3 D$ & DASAF-BNE \\
\hline netflux & Net surface heatflux & $\mathrm{W} \mathrm{m}^{-2}$ & $3 D$ & DASAF-BNE \\
\hline$q 2 m$ & Specific humidity at 2 msensible heat flux & $\mathrm{g} \mathrm{kg}^{-1}$ & $3 D$ & DASAF-BNE \\
\hline $\operatorname{shf}$ & Sensible heat flux & $W m^{-2}$ & $3 D$ & DASAF-BNE \\
\hline sst & Sea surface temperature & $\stackrel{\circ}{C}$ & $3 \mathrm{D}$ & DASAF-BNE \\
\hline swr & Short wave radiation & $\mathrm{W} \mathrm{m}^{-2}$ & $3 D$ & DASAF-BNE \\
\hline $\mathrm{t} 2 \mathrm{~m}$ & Air temperature at $2 \mathrm{~m}$ & 으 & $3 D$ & DASAF-BNE \\
\hline tau & Wind stress magnitude & $\mathrm{N} \mathrm{m}^{-2}$ & $3 D$ & DASAF-BNE \\
\hline taux & Zonal wind stress & $\mathrm{N} \mathrm{m}^{-2}$ & $3 \mathrm{D}$ & DASAF-BNE \\
\hline tauy & Meridional wind stress & $\mathrm{N} \mathrm{m}^{-2}$ & $3 D$ & DASAF-BNE \\
\hline ws & Wind speed at $10 \mathrm{~m}$ & $\mathrm{~m} \mathrm{~s}^{-1}$ & $3 D$ & DASAF-BNE \\
\hline precip & Daily precipitation rate & $\mathrm{mm}$ day $^{-1}$ & $3 D$ & DRA-BNE \\
\hline temp & Sea water potential temperature & $\stackrel{\circ}{C}$ & $4 \mathrm{D}$ & MAOP-BNE \\
\hline salt & Sea water salinity & psu & 4D & MAOP-BNE \\
\hline ssh & Sea surface height above geoid & $\mathrm{m}$ & $3 D$ & MAOP-BNE \\
\hline mlt & Mixed layer depth determined by temperature criteria & $\mathrm{m}$ & $3 D$ & MAOP-BNE \\
\hline $\mathrm{mlp}$ & Depth of potential density mixed layer & $\mathrm{m}$ & $3 D$ & MAOP-BNE \\
\hline $\mathrm{mls}$ & Mixed layer depth determined by salinity criteria & $\mathrm{m}$ & $3 D$ & MAOP-BNE \\
\hline net_heating & $\begin{array}{l}\text { Surface ocean heat flux coming through coupler and } \\
\text { mass transfer }\end{array}$ & $\mathrm{W} \mathrm{m}^{-2}$ & $3 \mathrm{D}$ & MAOP-BNE \\
\hline prho & Potential density referenced to $0 \mathrm{dbar}$ & $\mathrm{Kg} \mathrm{m}^{-3}$ & $4 \mathrm{D}$ & MAOP-BNE \\
\hline u & Seawater zonal velocity & $\mathrm{m} \mathrm{s}^{-1}$ & 4D & MAOP-BNE \\
\hline v & Seawater meridional velocity & $\mathrm{m} \mathrm{s}^{-1}$ & $4 \mathrm{D}$ & MAOP-BNE \\
\hline current_speed & Seawater velocity & $\mathrm{m} \mathrm{s}^{-1}$ & $4 \mathrm{D}$ & MAOP-BNE \\
\hline wt & Vertical current velocity & $\mathrm{m} \mathrm{s}^{-1}$ & 4D & MAOP-BNE \\
\hline tauy & Surface downward meridional stress & $\mathrm{N} \mathrm{m}^{-2}$ & $3 D$ & MAOP-BNE \\
\hline $\operatorname{taux}$ & Surface downward zonal stress & $\mathrm{N} \mathrm{m}^{-2}$ & $3 D$ & MAOP-BNE \\
\hline
\end{tabular}




\section{ACKNOWLEDGEMENTS}

H. L. V. Thanks to Coordenação de Aperfeiçoamento de Pessoal de Nível Superior - Brasil (CAPES) through the project Ciências do Mar II, AUXPE 1979/2014 and a pos doc grant to H. L. Varona. Finance Code 001, to TRIATLAS project (Tropical and South Atlantic climate-based marine ecosystem predictions for sustainable management), to Tropical Atlantic Interdisciplinary Laboratory on physical, biogeochemical, ecological and human dynamics (IJL TAPIOCA), and the Brazilian Research Network on Global Climate Change (Rede CLIMA).

\section{REFERENCES}

ADLER, R.F. et al. The Version-2 Global Precipitation Climatology Project (GPCP) Monthly Precipitation Analysis (1979-Present), Journal of Hydrometeorology, American Meteorological Society, 4 (6), 1147-1167, 2003. DOI: https://doi.org/10.1175/1525-7541(2003)004<1147:tvgpcp>2.0.co;2

CARTON, J. A.; GIESE, B. S. A Reanalysis of Ocean Climate Using Simple Ocean Data Assimila-tion (SODA), Monthly Weather Review, American Meteorological Society, 136 (8), 2999-3017, 2008. DOI: https://doi.org/10.1175/2007mwr1978.1

PIERCE, D. W. ncview: a netCDF visual browser, Scripps Institution of Oceanography, 2016. Available at $<$ http://meteora.ucsd.edu/pierce/ncview_home_page.html>. Accessed in 2021-07-01.

KUMAR, B. P. et al. TropFlux: air-sea fluxes for the global tropical oceans-description and evaluation, Climate Dynamics, Springer Science and Business Media LLC, 38 (7-8), 1521-1543, 2011. DOI: https://doi.org/10.1007/s00382-011-1115-0

SCHULZWEIDA, U. CDO user's guide. Hamburg: Climate data operators version 1.0.1. Max-Planck-Institute for Meteorology, 2006.2 Available at <https://src.fedoraproject.org/lookaside/pkgs/cdo/cdo.pdf/90a93037089dddf6f8919b9d6c30bff7/cdo.pdf >. Accessed in 2021-11-12.

VARONA, H. L. mNC: A tool for Oceanographers and Meteorologists to easily create their NetCDF files using Matlab (1.0), Zenodo, 2021a. DOI: https://doi.org/10.5281/zenodo.5572749

VARONA, H. L. CalcPlotAnomaly: Matlab function set for the calculation and plotting of anomalies (1.01), Zenodo, 2021b. DOI: https://doi.org/10.5281/zenodo.5576889

ZENDER, C. S. Analysis of self-describing gridded geoscience data with netCDF Operators (NCO), In Environmental Modelling \& Software, 23 (10-11), 1338-1342, Elsevier BV, 2008. DOI: https://doi.org/10.1016/j.envsoft.2008.03.004 